\section{ERCP and Balloon Dilation Is a Valuable Alternative to Surgical Biliodigestive Anastomosis in the Long Common Channel Syndrome in Childhood}

Choledochal cysts are a rare cause of hyperbilirubinemia in childhood. We report here a boy of two years of age with a long common channel syndrome, with multiple stenosis of the bile duct system and choledochal cysts, in whom endoscopic balloon dilation was able to restore biliary drainage.

The patient presented with a two-week history of nausea and emesis, followed by darkening of the urine and acholic feces. Serological tests for hepatitis were negative. Ultrasonography and CT disclosed dilation of the common bile duct to $13 \mathrm{~mm}$ and intrahe-

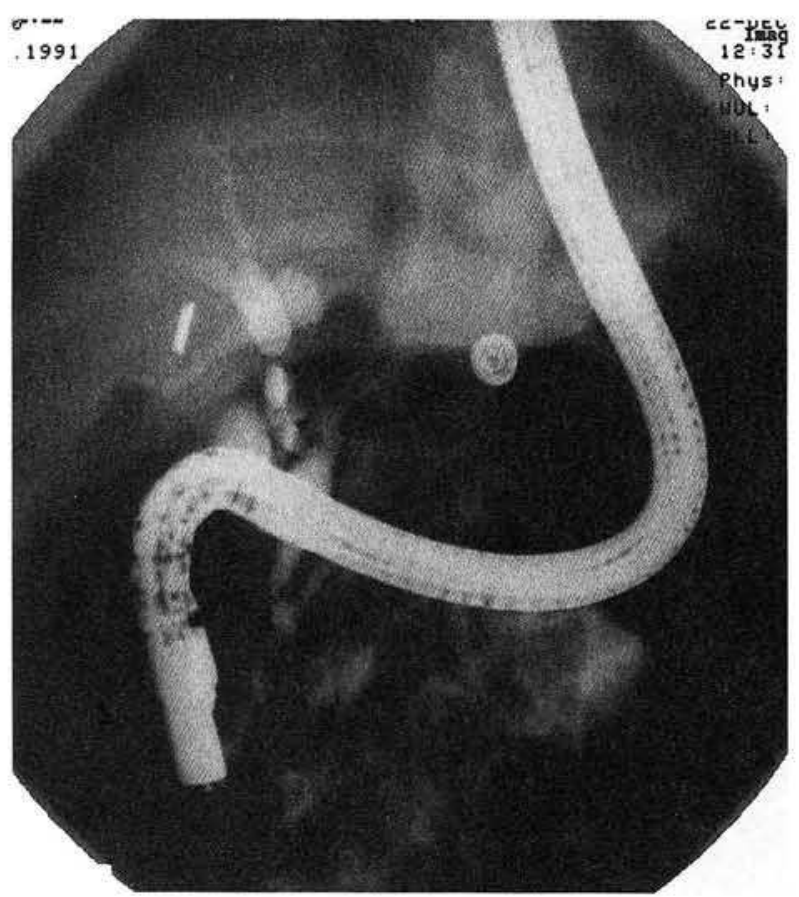

Figure 1: Multiple stenoses and cystic dilated segments of the common bile duct. patic cholestasis, confirming the diagnosis of a cystic long common channel syndrome. Increasing bilirubin levels and concomitant pancreatitis made it necessary to consider surgical intervention. Endoscopic retrograde cholangiopancreatography (ERCP) under general anesthesia showed a common channel $2 \mathrm{~cm}$ in length, three filiform stenoses of the common bile duct and prestenotic pseudocystic dilation of the extrahepatic bile duct (Figure 1). We decided to undertake balloon dilation of the obstructed segments. Via a guide wire, a $2-\mathrm{cm} 5-\mathrm{Fr}$ balloon was sucessfully placed in the narrowed segments of the common channel and then insufflated repeatedly. A dramatic increase of bile flow from the obstructed duct system ensued immediately (Figure 2). During the following days, declining cholestasis and an improvement in the pancreatitis were observed. Ten days after ERCP, all laboratory values were normal. The common bile duct remained slightly dilated $(9 \mathrm{~mm})$.

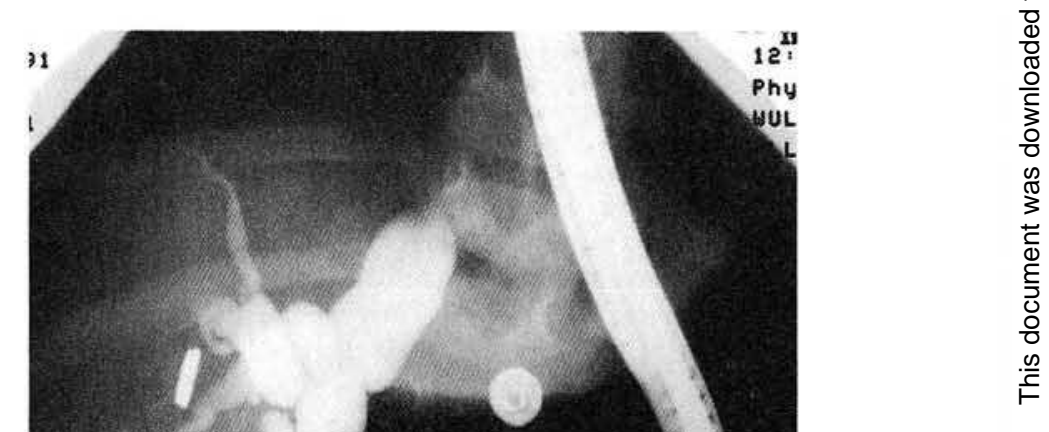


The boy was discharged without signs of cholestasis and pancreatitis. Follow-up examinations three month, six months, and one year after the ERCP showed normal liver values.

The long common channel syndrome has frequently been observed in Japan, but in Europe only a few cases have been reported (13). Some authors have suggested that a risk factor complicating cholangitis and pancreatitis is defective functioning of the sphincter of Oddi. This permits reflux of pancreatic juice into the bile duct system, thereby inducing an elevated pressure and thus promoting the development of choledochal cysts (4). Endoscopic sphincterotomy alone is rarely an adequate approach, since the common channel most often extends to the extraduodenal segment of the common bile duct. An alternative approach is repeated balloon dilation of the narrowed segments. Since the only alternative to this approach is surgical biliodigestive anastomosis, the procedure described above should be attempted first $(3,5)$.

\section{References}

1. Okada A, Nagoaka M, Kamata $S$, et al.: "Common channel syndrome": anomalous junction of the pancreatico-biliary ductal system. Kinderchirurgie 1981; 32: 144-151.

2. Schweizer P, Schweizer M: Pancreaticobiliary long common channel syndrome and congenital anomalous dilatation of the choledochal duct: study of 46 patients. Eur J Pediatr Surg 1993; 3: $15-21$.
3. Okada A, Oguchi Y, Kamata S, et al.: Common channel syndrome: diagnosis with endoscopic retrograde cholangiopancreatography and surgical management. Surgery 1983; 93: 634 642.

4. Van der Spuy S: Endoscopic retrograde cholangio-pancreatography (ERCP) in children. Endoscopy 1978; 10: 173-175.

5. Buckley A, Connon JJ: The role of ERCP in children and adolescents. Gastrointest Endosc 1990; 36: 369-372.

C. Sebestal, A. Schmid', P. Kier', R. Ruckserl,

E. Tiefengraber ${ }^{1}, H$. Rosen $^{2}$, K. Stelzhammer ${ }^{3}, K$. Geissler ${ }^{4}$, W. Hruby ${ }^{3}$, E. P. Horcher ${ }^{4}$, W. Hinterberger'

'Second Medical Department

${ }^{2}$ Department of Surgery

${ }^{3}$ Department of Radiology

${ }^{4}$ Department of Pediatric Surgery, Donauspital, Vienna, Austria

Corresponding Author

C. Sebesta, M.D.

Second Medical Department

Donauspital, Langobardenstraße 122

1220 Vienna

Austria

Fax: +43-1-2207611 